\title{
Longitudinal solitons in bunched beams
}

\author{
M. Blaskiewicz* and J. Wei \\ BNL, Upton, New York 11973, USA
}

A. Luque and H. Schamel

University of Bayreuth, 95440 Bayreuth, Germany

(Received 4 February 2004; published 28 April 2004)

\begin{abstract}
Stable, coherent, longitudinal oscillations have been observed in several accelerators. Within the context of perturbation theory, the beam parameters and machine impedance often suggest these oscillations should be Landau damped. When nonlinear effects are included, long-lived, stable oscillations become possible for low intensity beams. In this paper we report observations of stable humps in bunched beams and present a theoretical framework for their description. Implications for bunched beam stochastic cooling are considered.
\end{abstract}

DOI: 10.1103/PhysRevSTAB.7.044402

PACS numbers: 29.27.-a

\section{INTRODUCTION}

Solitary waves in the form of holes or humps have been observed in plasmas and coasting beams and the theory has been developed [1-6]. These solitons are stationary solutions of a coupled Vlasov-Poisson system.

For bunched beams below transition, the space charge force reduces the incoherent synchrotron frequency and, as such, is generally referred to as a defocusing impedance. Above transition space charge is focusing, leading to an increase in the incoherent synchrotron frequency. The change from focusing to defocusing at transition is an example of the mass conjugation theorem [4,7], and we will generally refer to focusing and defocusing impedances so that our results apply equally well above and below transition. Since we consider relativistic beams, the wall impedance is also included.

Solitons in bunched beams were suggested by observations of "rf activity" during bunched beam stochastic cooling studies in the SPS [8] and the Tevatron $[9,10]$. The rf activity was strong and extended to very high frequency, making the design of a cooling system difficult. A bunched beam stochastic cooling system for RHIC is in the design stage [11] and the possibility of a halo cooling system for the LHC has been considered [12]. It is therefore both timely and prudent to acquire a working knowledge of these phenomena. Before proceeding with the new material we review the coasting beam case.

Let $\theta$ denote machine azimuth and $\omega_{0}$ be the angular revolution frequency. We use the comoving phase $\phi=$ $\theta-\omega_{0} t$ as the longitudinal coordinate. Assume that the beam current $I(\phi, t)$ varies slowly in the comoving frame so that the longitudinal voltage per turn is given by

$$
V(\phi, t)=-\int W\left(\phi_{1}\right) I\left(\phi_{1}-\phi, t\right) d \phi_{1},
$$

*Electronic address: mmb@bnl.gov where $W(\phi)$ is the longitudinal wake potential, and we have assumed a short range wakefield to remove multiturn contributions to the voltage. This wakefield includes both the space charge piece as well as the contributions from bellows, transitions, etc. We have made the usual approximation that the frequency spread in the beam is small enough so that the locations of the impedance producing objects in the ring are irrelevant and only the sum of all the individual contributions matters. The equation of motion for a single particle is

$$
\frac{d^{2} \phi}{d t^{2}}=-\frac{\eta \omega_{0}^{2} q}{2 \pi \beta^{2} E} V(\phi, t)
$$

where $\eta=1 / \gamma_{T}^{2}-1 / \gamma^{2}$ is the frequency slip factor, $q$ is the charge per particle, $E$ is the central energy, and $\beta=v / c$. Set $p=d \phi / d t$ and introduce the phase space density $f(\phi, p, t)$, where $f d p d \phi$ is the number of particles in $d p d \phi$. The Vlasov equation for $f$ is then

$$
\frac{\partial f}{\partial t}+p \frac{\partial f}{\partial \phi}-\frac{\eta \omega_{0}^{2} q}{2 \pi \beta^{2} E} V(\phi, t) \frac{\partial f}{\partial p}=0,
$$

where

$$
V(\phi, t)=-q \omega_{0} \int W\left(\phi_{1}\right) d \phi_{1} \int f\left(\phi_{1}-\phi, p\right) d p .
$$

Soliton solutions are independent of time and nonlinear. When

$$
W(\phi)=W_{0} \Delta^{2} \operatorname{sgn}(\phi) \exp (-\Delta|\phi|),
$$

where $\Delta>0$ and $W_{0}$ are constants, the voltage is related to the current via

$$
V(\phi)-\Delta^{2} \frac{\partial^{2} V}{\partial \phi^{2}}=2 W_{0} \frac{\partial I}{\partial \phi} .
$$

For this case analytical progress is possible [4]. Certain other wakefields are tractable as well [2-4]. For the present case we will set $\Delta=0$ so that the coasting 


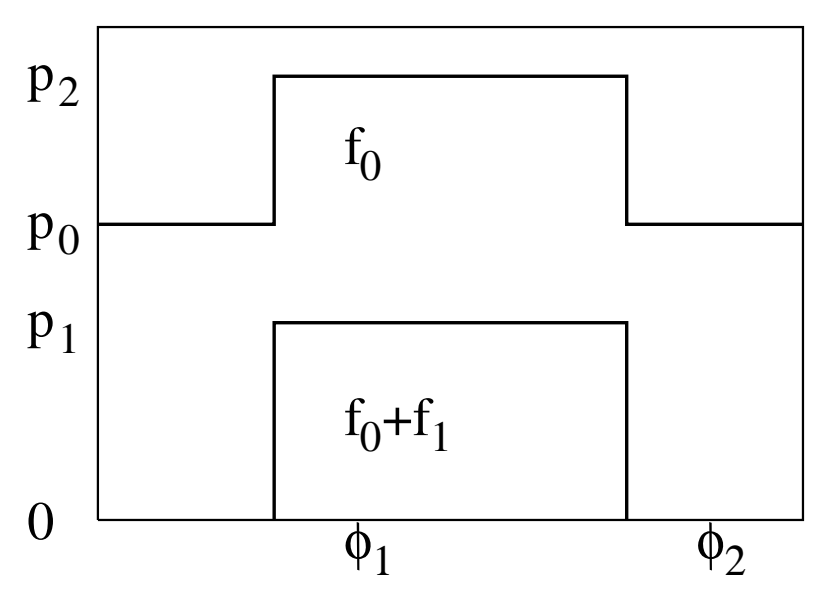

FIG. 1. Simple picture of a soliton in a coasting beam. The horizontal axis is $\phi$, the longitudinal position within the bunch. The vertical axis is $p=d \phi / d t$. The density is symmetric in $p$ and only the $p>0$ portion is shown.

beam Hamiltonian is approximated as

$$
H(\phi, p)=p^{2} / 2+k / 2 \int d p f(p, \phi) .
$$

The constant is given by

$$
k=\frac{2 \eta\left(q \omega_{0}\right)^{2} L}{\beta^{2} E},
$$

where $L$ is the net inductance. The net inductance satisfies $j \omega_{0} L=Z / n$, where $Z$ is the sum of the broadband wall impedance and the longitudinal space charge impedance.

For a round Gaussian beam with rms radius $\sigma$, in a round pipe of radius $b \gg \sigma$, the longitudinal space charge impedance is well approximated by

$$
\left.\frac{Z}{n}\right|_{\mathrm{sc}}=-j \frac{Z_{0}}{\beta \gamma^{2}} \ln \left(\frac{b}{1.5 \sigma}\right),
$$

where $\gamma=1 / \sqrt{1-\beta^{2}}$ is the Lorentz factor, $Z_{0}=377 \Omega$, and the derivation is given in the appendix.

Figure 1 shows a model due to Sacherer [13], in the frame comoving with the soliton. The piecewise constant, phase space density is either $0, f_{0}$, or $f_{0}+f_{1}$; and the distribution is independent of time. Since the phase space density is constant on contours of constant $H$, one obtains the equations $H\left(\phi_{1}, p_{1}\right)=H\left(\phi_{2}, 0\right)$ and $H\left(\phi_{1}, p_{2}\right)=$ $H\left(\phi_{2}, p_{0}\right)$. These may be combined to give

$$
p_{2}^{2}-p_{0}^{2}=p_{1}^{2}=2 k\left[f_{0}\left(p_{0}-p_{2}\right)-f_{1} p_{1}\right] .
$$

Taking $p_{0}, f_{0}$, and $p_{1}$ as input parameters yields $p_{2}=\sqrt{p_{0}^{2}-p_{1}^{2}} \quad$ and $\quad p_{1}^{2}=2 k\left[f_{0}\left(p_{0}-\sqrt{p_{0}^{2}-p_{1}^{2}}\right)-\right.$ $\left.f_{1} p_{1}\right]$. Taking $p_{1} \ll p_{0}$ and expanding the square root gives

$$
p_{1} \approx \frac{-2 k f_{1}}{1+k f_{0} / p_{0}}
$$

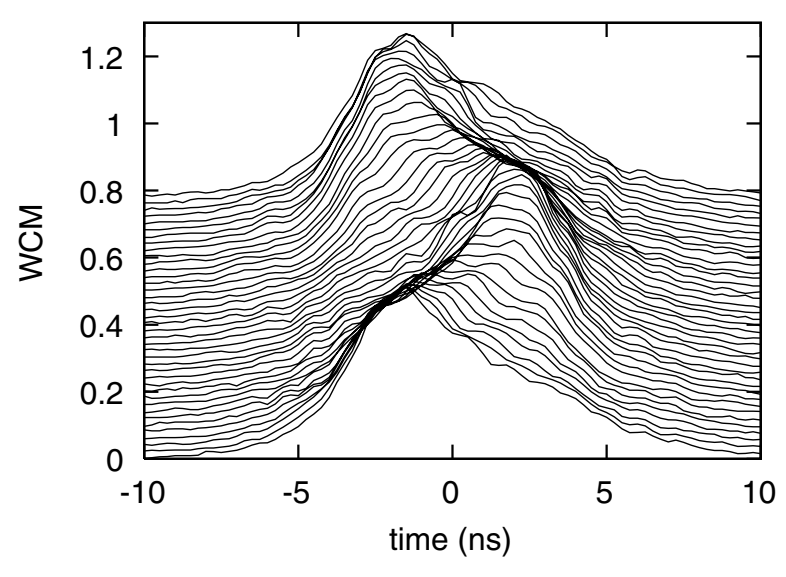

FIG. 2. Wall current monitor data for a freshly injected bunch.

If the correction term $k f_{0} / p_{0}$ is set to zero, the condition is identical to that for a phase space density of $f_{1}$ to self bunch. The change in the line density due to the soliton is $\approx-p_{1}^{2} / k$. For an inductive impedance above transition $k>0$. This results in a deficit of phase space density and one observes a hole in the line density. When $\Delta \neq 0$ the problem is more difficult. In a forthcoming publication [14], in which the delta effect is taken into account, we shall investigate the extent to which these predictions of the Sacherer model can be maintained. We go on to present some bunched beam data for the RHIC.

\section{DATA}

Long-lived coherence has been observed in the SPS $[8,12]$, the Tevatron [9,15], and now the RHIC [16]. Figure 2 shows a mountain range plot of the line density for freshly injected protons with $\gamma=25.9$. The amplitude of the coherent oscillation increased steadily, and Fig. 3 shows the same bunch, still at injection energy, 17 min later. Figures 4 and 5 show different bunches at

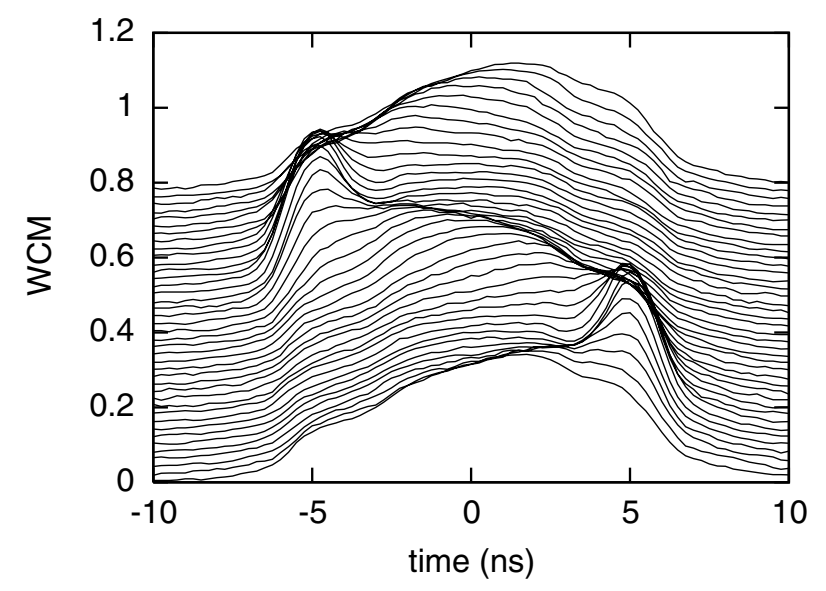

FIG. 3. Wall current monitor data at injection for the same bunch as in Fig. 2, but 17 min later. 


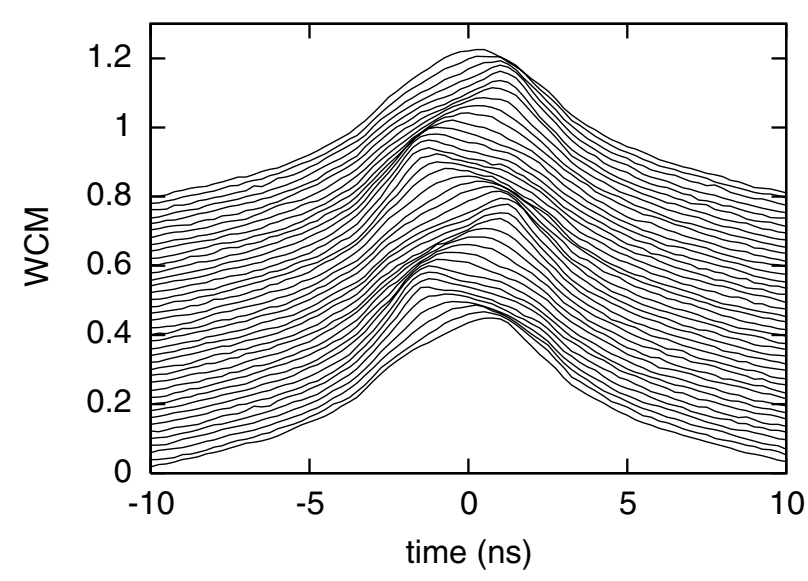

FIG. 4. Wall current monitor data for a bunch at the beginning of flattop.

flattop with $\gamma=107$. In all the cases shown, only the $28 \mathrm{MHz}$ accelerating cavities were operating and the total acquisition time was 4000 turns $\approx 50$ ms. RHIC's transition energy is $\gamma_{T}=23.8$, so all the data are above transition. All the data show a coherent oscillation which corresponds to a region of overdensity, or hump, in the longitudinal phase space. This behavior is commonplace in RHIC and we have never observed a stable hole. Measurements of RHIC's broadband impedance [17] give $Z / n=j(3 \pm 1) \Omega$ for the inductive wall contribution. The longitudinal space charge impedance at $\gamma=25.9$ is $Z / n=-j 1.3 \Omega$ and the space charge impedance becomes negligible at store. For all the data shown the coherent force leads to a reduction in the incoherent synchrotron frequency and as such it is defocusing, in the usual sense. We have also obtained data showing humps in deuteron beams with $\gamma=10.7<\gamma_{T}$ and $Z / n \approx-5 j \Omega$. Again, the deuterons show humps for impedances that are usually thought of as defocusing. Therefore, we always see humps with a defocusing impedance, which is just the reverse of

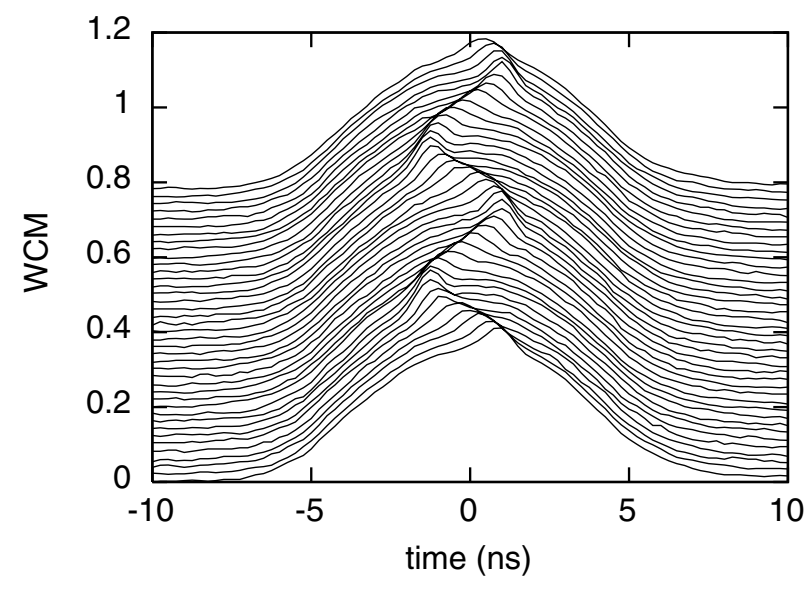

FIG. 5. Wall current monitor data for a different bunch at flattop. what one expects for coasting beams. We go on to consider the theory of solitons in bunched beams.

\section{BUNCHED BEAM MODEL}

For bunched beams we simply include the rf voltage in the coasting beam equations of motion. To simplify notation let $\phi$ denote the position in the bunch measured in units of rf radians, $\omega_{s, 0}$ denote the small amplitude angular synchrotron frequency, and use $s=\omega_{s, 0} t$ as the evolution variable. Let $\rho(\phi, s)$ be the normalized line density of the particles

$$
\int_{-\pi}^{\pi} d \phi \rho(\phi, s)=1
$$

The rf voltage is $V(t)=V_{\text {rf }} \sin \phi$ and $\omega_{\text {rf }}$ is the angular rf frequency. The total charge in the bunch is $Q$ which is assumed positive, and the effect of transition energy is included in the sign of the rf voltage with $V_{\text {rf }}>0$ below transition and $V_{\mathrm{rf}}<0$ above transition. Since solitons are long lived we neglect the effect of any synchronous phase. The equation of motion for $\phi$ is

$$
\frac{d^{2} \phi}{d s^{2}}+\sin \phi=\frac{L Q \omega_{\mathrm{rf}}^{2}}{V_{\mathrm{rf}}} \frac{\partial \rho(\phi, s)}{\partial \phi} .
$$

To simplify notation set

$$
\ell=-\frac{L Q \omega_{\mathrm{rf}}^{2}}{V_{\mathrm{rf}}}
$$

Space charge dominated beams below transition have $\ell>0$. For a steady state, matched bunch, a positive value of $\ell$ defocuses the beam and leads to an incoherent synchrotron frequency that is less than the synchrotron frequency for $\ell=0$. Set $p=d \phi / d s$ so that

$$
\frac{d p}{d s}=-\sin \phi-\ell \frac{\partial \rho(\phi, s)}{\partial \phi} .
$$

A drift-kick computer code has been written to simulate these equations of motion. Figure 6 shows simulation results for a short, roughly matched bunch that is displaced in phase from the stable fixed point. As is clear from the figure, a positive value of $\ell$ produces solitons of high density. Solitons in the RHIC accelerator always have $\ell>0$. However, for some ranges of the simulation parameters a hump can form with a focusing impedance. Next we present an analytic model of this phenomena.

\section{EQUATIONS OF MOTION}

Taking $p$ as the momentum coordinate and $\phi$ as the position coordinate, Eq. (3) follows from the Hamiltonian

$$
H(\phi, p, s)=p^{2} / 2+1-\cos \phi+\ell \rho(\phi, s) .
$$

Introduce the distribution function $f(\phi, p, s)$, where $f(\phi, p, s) d \phi d p$ is the fraction of particles in $d \phi d p$. 

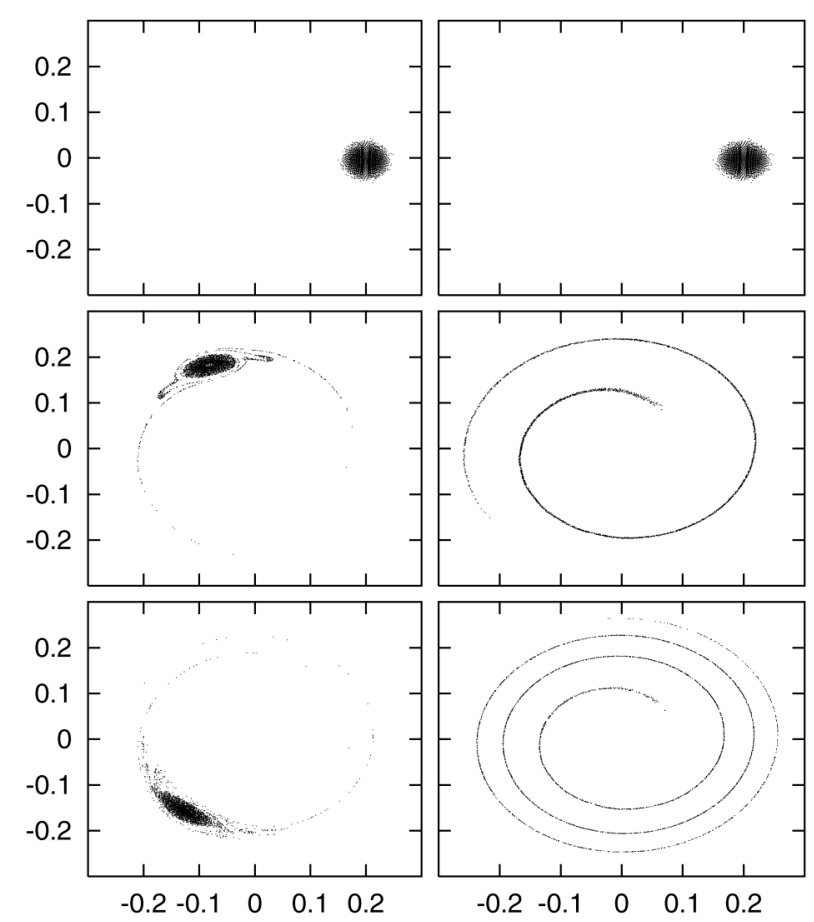

FIG. 6. Scatter plots of $p$ versus $\phi$ from longitudinal simulations with a sinusoidal rf voltage and a broadband impedance. The initial conditions (top panels) are identical and the only difference during the evolution is the sign of the broadband impedance. Effects of a defocusing impedance are shown on the left and those for a focusing impedance are shown on the right. Plots at 400 synchrotron periods (middle panels) and 800 synchrotron periods (bottom panels) clearly show that a defocusing impedance can lead to solitons.

The line density is then

$$
\rho(\phi, s)=\int d \phi_{1} d p_{1} \delta\left(\phi-\phi_{1}\right) f\left(\phi_{1}, p_{1}, s\right) .
$$

Consider a canonical transformation of Goldstein's [18] first type with a generator given by

$$
F_{1}(\phi, \Psi)=\frac{\phi^{2}}{2} \cot (\Psi+s)
$$

where $\Psi$ is the new position coordinate and $J$ is the new momentum coordinate. The old and new coordinates are related via

$$
p=\sqrt{2 J} \cos (\Psi+s), \quad \phi=\sqrt{2 J} \sin (\Psi+s) .
$$

These are rotating coordinates so both $J$ and $\Psi$ are "slow" variables as long as the bunch is not too long. Since the transformation is canonical $d p d \phi=d J d \Psi$. The Hamiltonian in the new variables is

$$
\begin{aligned}
& H_{1}(\Psi, J, s)=H+\left.\frac{\partial F_{1}}{\partial s}\right|_{\phi, \Psi} \\
=H-J & \\
= & -J+J \cos ^{2}(\Psi+s) \\
& +(1-\cos [\sqrt{2 J} \sin (\Psi+s)]) \\
& +\ell \int d \Psi_{1} d J_{1} f\left(\Psi_{1}, J_{1}, s\right) \delta[\sqrt{2 J} \sin (\Psi+s) \\
& \left.-\sqrt{2 J_{1}} \sin \left(\Psi_{1}+s\right)\right] .
\end{aligned}
$$

Assume that $f$ varies slowly compared with the sinusoids which is equivalent to assuming that the coherent frequency of the soliton is close to the small amplitude synchrotron frequency. Define the phase average of a generic function $h(J, \Psi, s)$ as

$$
\langle h(J, \Psi, s)\rangle_{s}=\frac{1}{2 \pi} \int_{0}^{2 \pi} h(J, \Psi, s) d s,
$$

with the slow variables held constant during the average. For the first line in Eq. (7)

$$
-J+\left\langle J \cos ^{2}(\Psi+s)\right\rangle_{s}+1-\langle\cos [\sqrt{2 J} \sin (\Psi+s)]\rangle_{s}=-J / 2+1-\mathrm{J}_{0}(\sqrt{2 J}) \equiv \alpha(J) \approx-J^{2} / 16,
$$

where $J_{0}(x)$ is the Bessel function, and we have introduced $\alpha(J)$ both for convenience and to allow for other forms of $\mathrm{rf}$ voltage. Averaging the Hamiltonian in (6) gives

$$
H_{1}=\alpha(J)+\ell \int d \Psi_{1} d J_{1} f\left(\Psi_{1}, J_{1}, s\right)\left\langle\delta\left[\sqrt{2 J} \sin (\Psi+s)-\sqrt{2 J_{1}} \sin \left(\Psi_{1}+s\right)\right]\right\rangle_{s} .
$$

To perform the phase average of the delta function notice

$$
\sqrt{2 J} \sin (\Psi+s)-\sqrt{2 J_{1}} \sin \left(\Psi_{1}+s\right)=\sqrt{2 J+2 J_{1}-4 \sqrt{J J_{1}} \cos \left(\Psi-\Psi_{1}\right)} \cos (s+\xi) \equiv R \cos (s+\xi),
$$

where $\xi$ is a phase that depends on $J, J_{1}, \Psi$, and $\Psi_{1}$. And

$$
\langle\delta[R \cos (s+\xi)]\rangle_{s}=\left.\int_{0}^{2 \pi} \frac{d s}{2 \pi} \delta[R \cos (s+\xi)]\right|_{R, \xi, \text { fixed }}=\int_{-\pi}^{\pi} \frac{d s}{2 \pi} \delta[R \cos (s)]=\frac{1}{\pi R} .
$$

The last steps follow since $\xi$ and $R$ are slow, and the delta function in the integrand on the right is nonzero only when $s= \pm \pi / 2$. The Hamiltonian becomes

$$
H_{1}=\alpha(J)+\frac{\ell}{\pi} \int d \Psi_{1} d J_{1} \frac{f\left(\Psi_{1}, J_{1}, s\right)}{\sqrt{2 J+2 J_{1}-4 \sqrt{J J_{1}} \cos \left(\Psi-\Psi_{1}\right)}}
$$


The equations of motion for $\Psi$ and $J$ are

$$
\frac{d \Psi}{d s}=\frac{\partial H_{1}}{\partial J}, \quad \frac{d J}{d s}=-\frac{\partial H_{1}}{\partial \Psi} .
$$

The corresponding Vlasov equation is

$$
\frac{\partial f}{\partial s}+\frac{\partial H_{1}}{\partial J} \frac{\partial f}{\partial \Psi}-\frac{\partial H_{1}}{\partial \Psi} \frac{\partial f}{\partial J}=0 .
$$

We seek uniformly rotating solutions (i.e., the solitons),

$$
f(\Psi, J, s)=g(\Psi+\tilde{r} s, J),
$$

where $\tilde{r}$ is a constant. Inserting this functional form in the Vlasov equation gives

$$
\left[\tilde{r}+\frac{\partial H_{1}}{\partial J}\right] \frac{\partial g}{\partial \Psi}-\frac{\partial H_{1}}{\partial \Psi} \frac{\partial g}{\partial J}=0,
$$

where $g=g(\Psi, J)$ is a function of two variables. For some purposes it is useful to transform back to Cartesian phase space coordinates.

$$
A=\sqrt{2 J} \sin \Psi, \quad B=\sqrt{2 J} \cos \Psi .
$$

This is a canonical transformation so the density $g(A, B)$ satisfies

$$
\frac{\partial K}{\partial B} \frac{\partial g}{\partial A}-\frac{\partial K}{\partial A} \frac{\partial g}{\partial B}=0
$$

with the new Hamiltonian

$$
K=R(A, B)+V(A, B),
$$

where the single particle motion is generated by

$$
R(A, B)=\alpha\left(\left[A^{2}+B^{2}\right] / 2\right)+\tilde{r}\left(A^{2}+B^{2}\right) / 2,
$$

and collective effects are due to

$$
V(A, B)=\frac{\ell}{\pi} \int d A_{1} d B_{1} \frac{g\left(A_{1}, B_{1}\right)}{\sqrt{\left(A-A_{1}\right)^{2}+\left(B-B_{1}\right)^{2}}}
$$

\section{APPROXIMATE SOLUTIONS}

For physical solutions of Eq. (12), local extrema of $g$ occur at stable fixed points of $K$. Additionally, contours of constant values of $g$ and $K$ coincide. Suppose we have a situation like that shown in the bottom left panel of Fig. 6 . Choose the $A$ axis to pass through the center of the soliton so it is centered at $A=A_{0}, B=0$. We will consider solutions for which this is a fixed point of both $R$ and $V$. In the vicinity of the fixed point the leading order approximation to the single particle generator is $R(A, B)=R_{0}+\lambda\left(A-A_{0}\right)^{2}$, where $\lambda \approx-A_{0}^{2} / 16$ for sinusoidal rf. To this order $\partial R / \partial A=2 \lambda\left(A-A_{0}\right)$ and $\partial R / \partial B=0$. In this approximation the curvature of the soliton apparent on the left side of Fig. 6 will be absent, but for solitons of small angular extent, the results should be good.

If one could find a class of functions $g(A, B)$ which were constant on elliptical contours, and yielded linear coherent forces within the beam, the Vlasov equation would reduce to an algebraic equation among the various parameters. Much of the relevant mathematics is the same as for transverse space charge [19] but will be reproduced here for completeness. First, notice that Eq. (15) is the electrostatic potential of a two dimensional charge sheet in the plane containing the sheet. Let us add a third dimension $z$.

The generalization of Eq. (15) is

$$
V(A, B, z)=\frac{\ell}{\pi} \int d A_{1} d B_{1} d z_{1} \frac{g\left(A_{1}, B_{1}, z_{1}\right)}{\sqrt{\left(A-A_{1}\right)^{2}+\left(B-B_{1}\right)^{2}+\left(z-z_{1}\right)^{2}}},
$$

which satisfies the equation

$$
\left(\frac{\partial^{2}}{\partial A^{2}}+\frac{\partial^{2}}{\partial B^{2}}+\frac{\partial^{2}}{\partial z^{2}}\right) V(A, B, z)=-4 \lg (A, B, z) .
$$

Consider distributions of the form,

$$
g(A, B, z)=\frac{n\left(A^{2} / a^{2}+B^{2} / b^{2}+z^{2} / c^{2}\right)}{a b c},
$$

and define

$$
T(A, B, z, \tau)=\frac{A^{2}}{a^{2}+\tau}+\frac{B^{2}}{b^{2}+\tau}+\frac{z^{2}}{c^{2}+\tau} .
$$

The field gradients are given by

$$
\begin{aligned}
\frac{\partial V}{\partial A}= & -2 \ell A \int_{0}^{\infty} d \tau \frac{n[T(A, B, z, \tau)]}{\left(a^{2}+\tau\right)} \\
& \times\left[\left(a^{2}+\tau\right)\left(b^{2}+\tau\right)\left(c^{2}+\tau\right)\right]^{-1 / 2},
\end{aligned}
$$

$$
\begin{aligned}
\frac{\partial V}{\partial B}= & -2 \ell B \int_{0}^{\infty} d \tau \frac{n[T(A, B, z, \tau)]}{\left(b^{2}+\tau\right)} \\
& \times\left[\left(a^{2}+\tau\right)\left(b^{2}+\tau\right)\left(c^{2}+\tau\right)\right]^{-1 / 2},
\end{aligned}
$$

$$
\begin{aligned}
\frac{\partial V}{\partial z}= & -2 \ell z \int_{0}^{\infty} d \tau \frac{n[T(A, B, z, \tau)]}{\left(c^{2}+\tau\right)} \\
& \times\left[\left(a^{2}+\tau\right)\left(b^{2}+\tau\right)\left(c^{2}+\tau\right)\right]^{-1 / 2} .
\end{aligned}
$$

Substituting Eqs. (20)-(22) in (17), and integrating by parts proves that the solution works up to the boundary condition terms. To prove the boundary conditions assume that $n(T)=0$ for $T>T_{0}$, and that $n(T) \leq n_{0}$ for all $T$. Assume $B^{2} / b^{2}>T_{0}$, then 


$$
\left|\frac{\partial V}{\partial B}\right| \leq|2 B \ell| \int_{B^{2} / T_{0}}^{\infty} n_{0} \tau^{-5 / 2} d \tau=\frac{4 n_{0} \ell}{3 T_{0}^{3 / 2} B^{2}}
$$

Next consider the potential in the $z=0$ plane

$$
V(A, B, 0)=\frac{\ell}{\pi} \int d A_{1} d B_{1} d z_{1} \frac{g\left(A_{1}, B_{1}, z_{1}\right)}{\sqrt{\left(A-A_{1}\right)^{2}+\left(B-B_{1}\right)^{2}+z_{1}^{2}}} .
$$

which goes to zero as $B \rightarrow \infty$. Letting $A \rightarrow \infty$ yields the same limit and proves the boundary conditions.

As $c \rightarrow 0$ we obtain a charge sheet with the two dimension potential

$$
V(A, B)=\frac{\ell}{\pi} \int d A_{1} d B_{1} \frac{1}{\sqrt{\left(A-A_{1}\right)^{2}+\left(B-B_{1}\right)^{2}}} \int d z_{1} g\left(A_{1}, B_{1}, z_{1}\right),
$$

where the last integral is independent of $c$.

For distributions where $n(T)$ is a constant for $T<T_{0}$ and zero otherwise, the gradients (20)-(22) are linear functions in the region where $n \neq 0$. Integrating over the $z$ dimension yields the class for functions needed for the solution of the Vlasov equation

$$
g(A, B)= \begin{cases}\frac{3}{2 \pi a b} \sqrt{1-\left(A-A_{0}\right)^{2} / a^{2}-B^{2} / b^{2}} & \text { if }\left(A-A_{0}\right)^{2} / a^{2}+B^{2} / b^{2}<1, \\ 0 & \text { otherwise, }\end{cases}
$$

where $a$ and $b$ are parameters, and we have shifted the center of the distribution to the stable fixed point.

In the region where $g \neq 0$ the coherent forces are

$$
\begin{gathered}
\frac{\partial V}{\partial A}=-\left(A-A_{0}\right) \frac{3 \ell}{2 \pi} \int_{0}^{\infty} d \tau\left(b^{2}+\tau\right)^{-1 / 2}\left(a^{2}+\tau\right)^{-3 / 2} \tau^{-1 / 2} \\
\frac{\partial V}{\partial B}=-B \frac{3 \ell}{2 \pi} \int_{0}^{\infty} d \tau\left(b^{2}+\tau\right)^{-3 / 2}\left(a^{2}+\tau\right)^{-1 / 2} \tau^{-1 / 2}
\end{gathered}
$$

With Eqs. (25)-(27) the Vlasov equation (12) is equivalent to

$$
a^{2}\left(2 \lambda+V_{A A}\right)=b^{2} V_{B B},
$$

where we have introduced the notation $V_{A A}=$ $\partial^{2} V / \partial A^{2}\left(A=A_{0}, B=0\right)$.

The potential $V(A, B)$ is "rounder" than the distribution $g(A, B)$. More explicitly, if $a<b$ then

$$
\frac{a^{2}}{b^{2}} \frac{V_{A A}}{V_{B B}}<1
$$

We have verified this via numerical calculation of the various integrals [20]. Intuitively, notice that $V(A, B)$ is the convolution of $g(A, B)$ with a cylindrically symmetric function.

Generally, one has $V_{A A}=-\left(\ell / a^{3}\right) G_{a}(a / b)$ and $V_{B B}=$ $-\left(\ell / a^{3}\right) G_{b}(a / b)$. Define $r=a / b$

$$
\begin{aligned}
G_{a}(r) & =\frac{3 r^{3}}{2 \pi} \int_{0}^{\infty} d \tau\left[(1+\tau)\left(r^{2}+\tau\right)^{3} \tau\right]^{-1 / 2} \\
& =3 r^{3} \sum_{n=1}^{\infty} n\left[\frac{(2 n-1) ! !}{2^{n} n !}\right]^{2}\left(1-r^{2}\right)^{n-1}
\end{aligned}
$$

$$
\begin{aligned}
G_{b}(r) & =\frac{3 r^{3}}{2 \pi} \int_{0}^{\infty} d \tau\left[\left(r^{2}+\tau\right)(1+\tau)^{3} \tau\right]^{-1 / 2} \\
& =(3 / 2) r^{3} \sum_{n=0}^{\infty}\left[\frac{(2 n-1) ! !}{2^{n} n !}\right]^{2}\left(1-r^{2}\right)^{n}-r^{2} G_{a}(r) .
\end{aligned}
$$

The summations are combinations of various elliptic integrals [21].

Equation (28) reduces to

$$
-\frac{2 a^{3} \lambda}{\ell}=G_{b}(r) / r^{2}-G_{a}(r) \approx-0.77 r \ln (r) /(1+r),
$$

where the approximate expression is accurate with a few percent for $0<r<10$. The right side of this equation is plotted as a function of $r$ in Fig. 7. The maximum value of 0.21224 occurs for $r=0.26982$. Figure 8 shows the individual $G_{a}$ and $G_{b}$. For $r>0.4, r^{2} G_{a} / G_{b} \approx r^{1 / 2}$, in

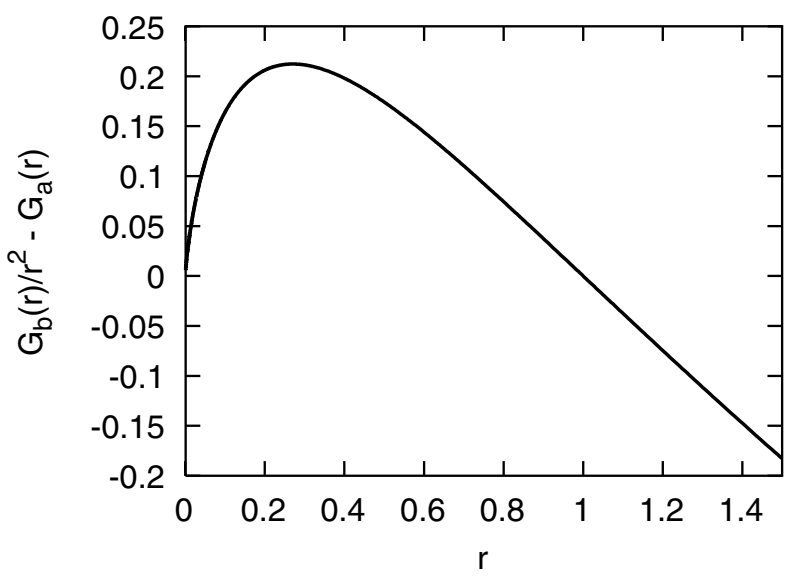

FIG. 7. Right-hand side of Eq. (32) as a function of $r=a / b$. 


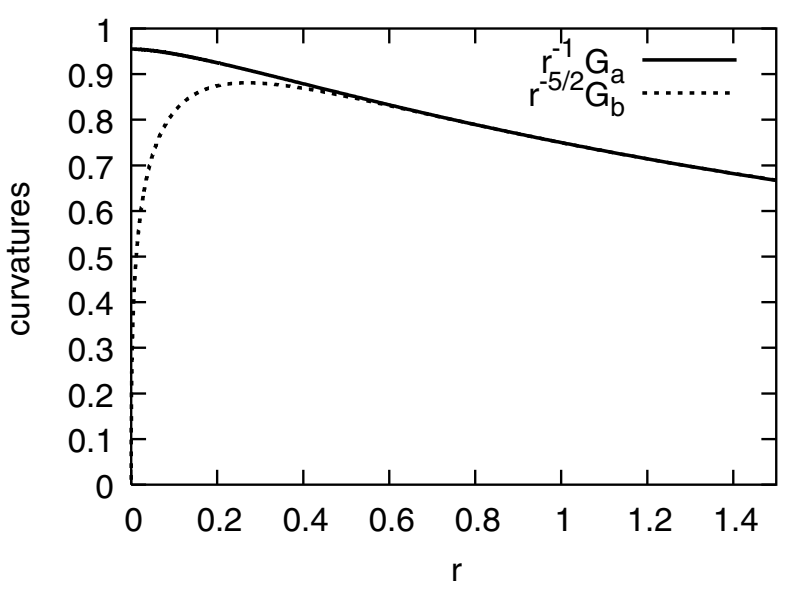

FIG. 8. Plots of the curvature functions scaled by selected powers of $r$.

accord with inequality (29). Notice that any $r>0$ is permissible. For $r<1$ the soliton is due to a defocusing impedance while a focusing impedance is required for $r>1$.

When a background beam is present, as shown in Figs. 2-5, analytic solutions of Eq. (12) have not been found. In the next section we present some numerical results.

\section{SELF-CONSISTENT NUMERICAL SOLUTIONS}

Equation (12) implies that contours of constant $g$ coincide with those of constant $K$. However, this does not imply that there always exists a single function $G(K)$ such that $g(A, B)=G[K(A, B)]$. To see this consider Fig. 9 which shows the Hamiltonian through a line containing both the origin and the center of the soliton for both

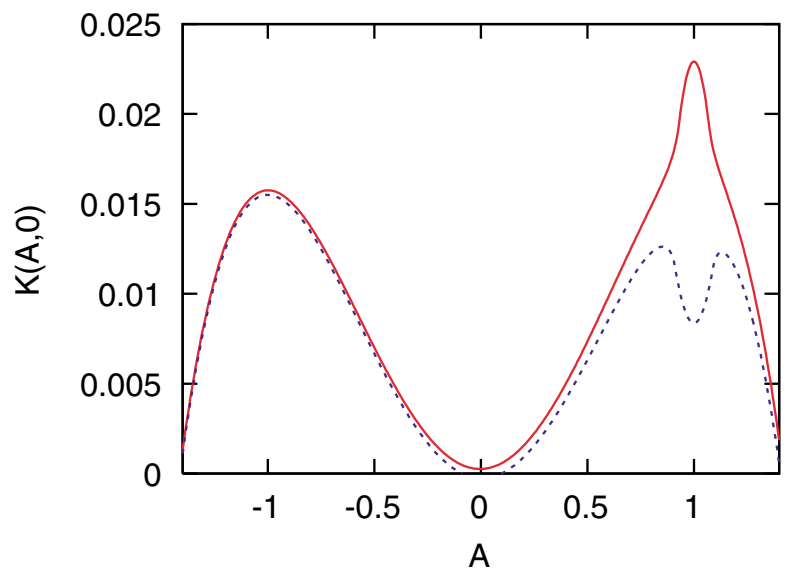

FIG. 9. (Color) Schematic plot of $K(A, B=0)$. The solid red line corresponds to a defocusing impedance with $\ell>0$ and the soliton centered at $A=1, B=0$. The blue dashed line corresponds to a focusing impedance with $\ell<0$ and the soliton centered at $A=1, B=0$. positive (solid red line) and negative (blue dashed line) values of $\ell$. For the moment consider the defocusing (solid red line) case, which has $\ell>0$. There are four values of $A$ for which $K=0.01$ but the density $g(A, B)$ need not have the same value at all four points. The key is the separatrix, on which $K=K_{s}$, which is shown in Fig. 10.

In each of the three regions bounded by the separatrix one is free to choose a different functional form $g=$ $G_{i}(K)$. There are limitless possibilities but we take the fairly simple prescription

$$
g(A, B)=C_{4} \begin{cases}G_{1}(x)=C_{0} x^{2}+C_{1}, & \text { in region } 1, \\ G_{2}(x)=C_{1}+\sqrt{C_{2}}-x-\sqrt{C_{2}}, & \text { in region } 2, \\ G_{3}(x)=C_{1}-\sqrt{C_{2}}-x+\sqrt{C_{2}}, & \text { in region } 3,\end{cases}
$$

with $x=K-K_{s}$, and the $C_{1}, \ldots, C_{4}$ are constants. With $g$ given by (33) the contours of constant $g$ and $K$ coincide, satisfying the Vlasov equation. However, the Hamiltonian depends on the density yielding the additional constraint

$$
\begin{aligned}
K(A, B)= & R(A, B) \\
& +\frac{\ell}{\pi} \int d A_{1} d B_{1} \frac{g\left(A_{1}, B_{1}\right)}{\sqrt{\left(\bar{A}-\overline{A_{1}}\right)^{2}+\left(\bar{B}-\overline{B_{1}}\right)^{2}}},
\end{aligned}
$$

where $R(A, B)$ is the single particle generator defined in Eq. (14).

We obtain self-consistent solutions for $\ell>0$ using the following procedure:

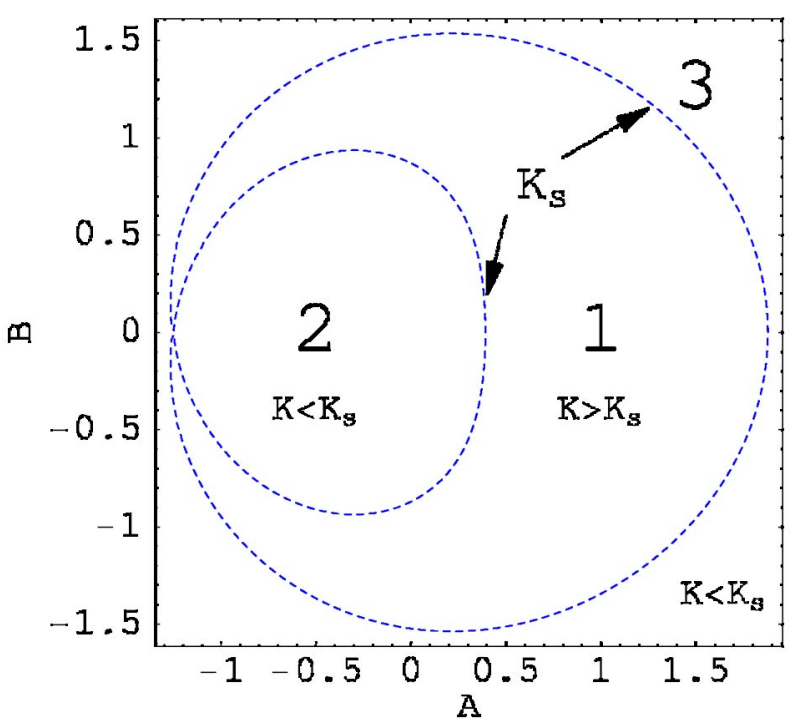

FIG. 10. (Color) Schematic contour plot of $K(A, B)$ with $\ell>0$ and a soliton present. The dashed line represents the separatrix, $K=K_{s}$. The particles are divided into three different groups: particles that belong to the soliton (region 1), particles inside the inner separatrix (region 2), and particles outside the outer separatrix (region 3). 
(1) Choose $\tilde{r}$. Set $n=0$. Choose initial parameters $C_{0}^{0}$, $C_{1}$, and $C_{2}$. Set up an initial line density $g^{0}(A, B)$ which is symmetric around $B=0$.

(2) Use $g^{n}(A, B)$ to calculate $K^{n}(A, B)$ through (34). Find the separatrix on which $K^{n}(A, B)=K_{s}^{n}$. In region $1, K^{n}(A, B)$ is maximum at the stable fixed point $\left(A=A_{0}^{n}, B=0\right)$ and minimum at the unstable fixed point. Both fixed points always lie on the line $B=0$.

Define the electrostatic amplitude $\Psi^{n}=K^{n}(O)-$ $K^{n}(X)$ where $O$ and $X$ denote the stable and unstable fixed points, respectively.

(3) If $n>0$ change $C_{0}$ through the formula

$$
C_{0}^{n+1}=C_{0}^{n}\left(1-\alpha \frac{\Psi^{n}-\Psi^{n-1}}{\Psi^{n}}\right),
$$

where $\alpha$ is an input parameter.

If $n=0$ use $C_{0}^{1}=C_{0}^{0}$.

(4) Calculate $g^{n+1}$ using Eq. (33) with $K^{n}(A, B)$ and $C_{0}^{n}$. Calculate the global normalization constant $C_{4}$ and increase $n$.

(5) Repeat the iteration steps (2)-(4) until $g(A, B)$ converges.

The variation in $C_{0}$, defined through Eq. (35), greatly increases the initial parameter space leading to interesting solutions; typically, $\alpha \sim 1$. Figure 11 shows the evolution of the density as the iteration proceeds.

Depending on the initial parameters, a large range of stable solutions can be obtained. An outcome of this numerical procedure is that there is a definite relationship between $\tilde{r}$ and the converged quantities $C_{0}$ and $\Psi$, denoted by $\Psi=\Psi\left(C_{0}, \tilde{r}\right)$ shown in Fig. 12 . It can be considered as a nonlinear dispersion relation (as in the standard soliton cases [1-4]), since it relates the soliton amplitude $\Psi$ and the trapping parameter $C_{0}$ with the phase speed $\tilde{r}$. As seen from (33), $C_{0}$ can be expressed by

$$
C_{0}=\frac{g(O)-g(X)}{N \Psi^{2}}
$$

where $g(0)[g(X)]$ is the distribution function at the stable (unstable) fixed point. It is clear that $C_{0}$ is positive for a

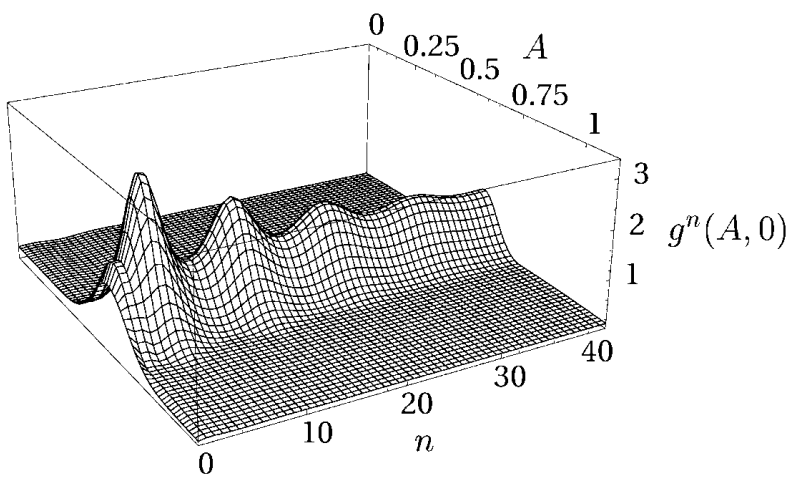

FIG. 11. Evolution of the density along $B=0$ during the iteration. Convergence is reached after about 30 iterations.

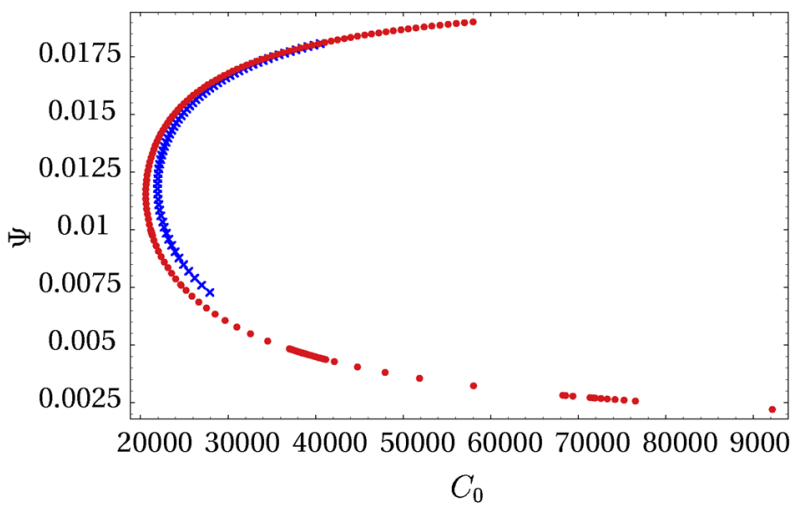

FIG. 12. (Color) Relationship between $C_{0}$ and the soliton potential, $\Psi$. The red circles (outer curve) correspond to $\tilde{r}=$ 0.025 and the blue crosses (inner curve) correspond to $\tilde{r}=$ 0.030 .

hump in region 1 of Fig. 10, zero for a flattopped and negative for a notch, and hence $C_{0}$ reflects the status of trapped particles. We learn from Fig. 12 that $C_{0}$ is strictly positive, corresponding to a hump (hot spot), and that there are two different values for $\Psi$ (for a given $\tilde{r}$ and $C_{0}$ ). For the larger $\Psi$ the majority of particles is residing in the hot spot (as in Fig. 13), whereas for the smaller value of $\Psi$ the particles are mostly contained in the background.

Figure 13 shows the phase space density and accompanying line density for a bunch with most of the beam trapped in the soliton, while Fig. 14 shows a small soliton. Both solutions have $\ell=0.01$ and $A_{0}=0.025$ showing the broad range of stable solutions that are possible for the same bunch charge and machine impedance. This is qualitatively consistent with Figs. 2-5. In the next section we will compare the results of the iterative solutions with simulations and with the approximate analytic solution.

\section{COMPARISON WITH SIMULATIONS}

Longitudinal simulations were carried out with the update equations:

$$
\begin{gathered}
p_{n+1}=p_{n}-\Delta \sin \phi_{n}+\Delta F_{c}\left(\phi_{n}\right), \\
\phi_{n+1}=\phi_{n}+\Delta p_{n+1},
\end{gathered}
$$

where $\Delta \sim 2 \pi / 1000$ is the update in $s$.

The coherent force $F_{c}(\phi)$ was obtained by (i) using linear interpolation to allocate the particle charges to a fine grid, (ii) smoothing the finely gridded data, (iii) applying a centered difference numerical differentiation, and (iv) using linear interpolation to get the forces.

For some of the simulations all the test particles had the same charge and for others the values of the test charge differed with the particle [22]. All particles had the same charge to mass ratio. In all cases the force of particle $i$ on particle $j$ was equal and opposite to the force of particle $j$ on particle $i$. Since the simulation is in one dimension 

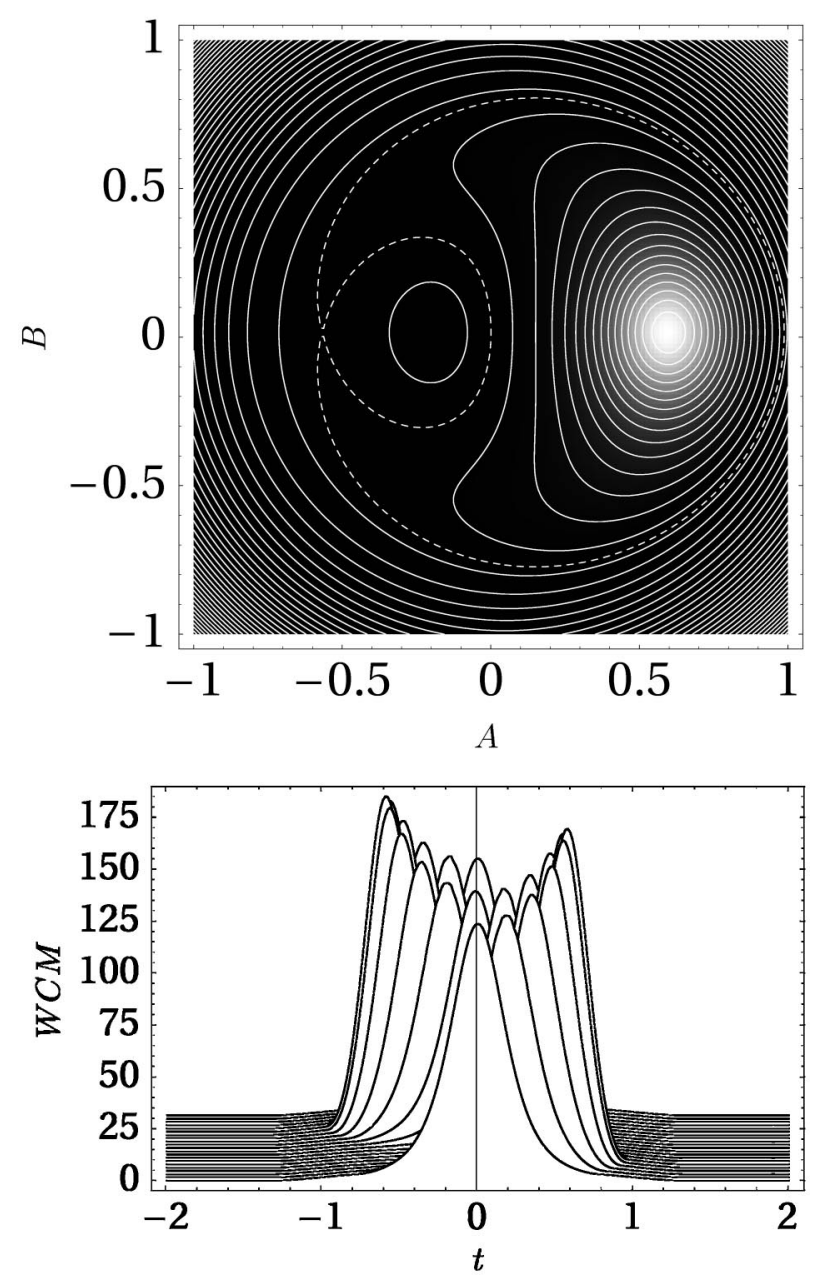

FIG. 13. (a) Phase space density (gray scale) and contours of constant energy $K(A, B)$ (particle trajectories). (b) Simulated wall current monitor data. The solution was obtained with $\alpha=$ $0.3, \tilde{r}=0.025, C_{1}=0.1, C_{3}=0.005, \ell=0.01, C_{0}=21975$, and $\Psi=0.014$. This solution consists of a large soliton with little background, i.e., almost all particles are trapped in the soliton.

there were no problems associated with thermalization effects so that having a range of particle charges produced no secular evolution.

Figure 15 compares the simulations to the analytic formula (32). The initial conditions were set up using Eq. (32) although a small amount of curvature was included by using the mapping $\phi=A \cos (B / A), \quad p=$ $A \sin (B / A)$. The simulation parameters are shown in Table I. Simulations 3 and 5 have $\ell<0$ while the others have $\ell>0$. For $\ell>0$ one has $a<b$ and all the simulations but 7 show no filamentation. Simulations 3 and 5 both show some filamentation. Examining the blue, dashed curve in Fig. 9 suggests an explanation. The curves in Fig. 9 were obtained from Eq. (13) using $V(A, B)$ numerically integrated from a solution of Eq. (32). The solutions of (32) had $\sqrt{a b}=0.08$ and $\ell= \pm 8 \times 10^{-4}$. For $\ell>0, a / b=0.833$; for $\ell<0, a / b=1.33$. Consider
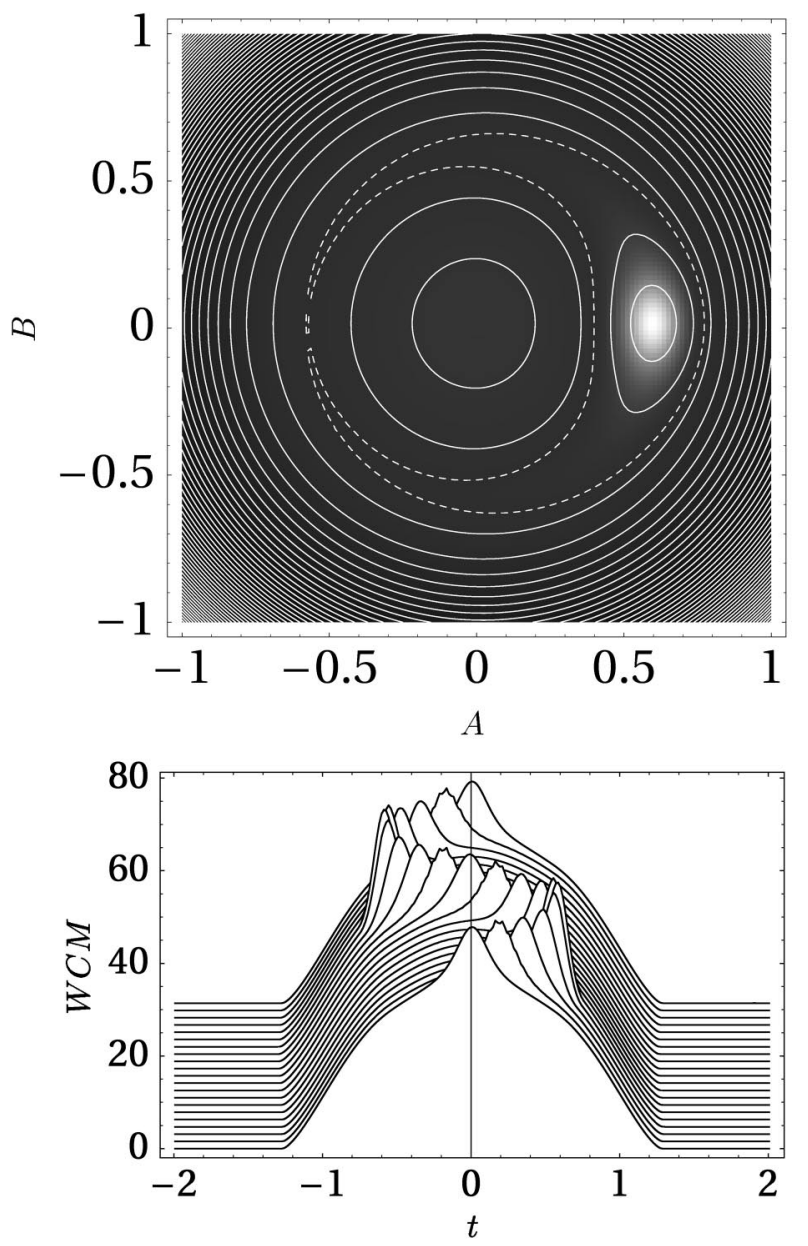

FIG. 14. (a) Phase space density (gray scale) and contours of constant energy $K(A, B)$ (particle trajectories). (b) Simulated wall current monitor data. The solution was obtained with $\alpha=$ 1.2, $\tilde{r}=0.025, C_{1}=0.1, C_{3}=0.005, \ell=0.01, C_{0}=92191$, and $\Psi=0.0022$. This solution has comparable density in the soliton and the background.

the potential difference between the edge of the soliton and the separatrix. For $\ell>0$ this value was 5.1 times larger than its value in the $\ell<0$ case. In other words, with all else equal, $\ell>0$ solitons make deeper buckets than $\ell<0$ solitons. These simulations confirm the accuracy of Eq. (32) for isolated solitons. Next we consider the utility of this equation for the case when a background beam is present.

Figure 16 shows simulation results for a soliton with a background beam. To obtain the initial conditions we used a modified form of Eq. (32):

$$
-\frac{2 a^{3} \lambda}{\chi^{\ell}}=G_{b}(r) / r^{2}-G_{a}(r) \approx-0.77 r \ln (r) /(1+r),
$$

where $\chi$ was the fraction of the total bunch charge in the hump, and $\ell$ is calculated using to total charge in the bunch. The parameters $a, r$, and $\lambda$ were calculated using 

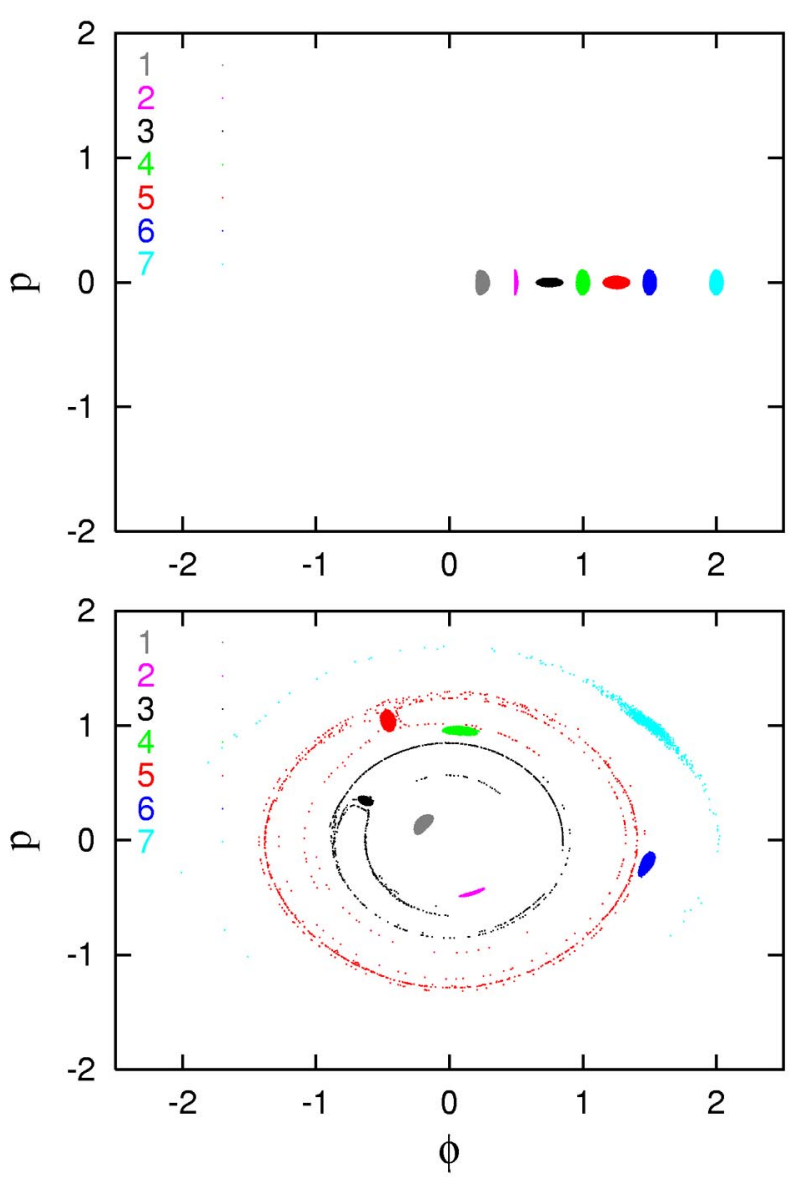

FIG. 15. (Color) Simulations using update equations (37) and (38) for isolated solitons. The initial states (top panel) are centered on $p=0$ and are indexed with increasing values of $\phi$. The initial values of $a, b, A_{0}$, and $\ell$ obey Eq. (32), with a slight modification for curvature. In each simulation all the test particles had the same charge. Plots of the final phase space are shown on the bottom. Simulation 2 was run for 1000 synchrotron oscillations while the others ran for 100 synchrotron oscillations.

the soliton parameters. In this case we used initial conditions with the macroparticles evenly spaced on a rectangular grid, and each macroparticle was assigned a charge proportional to the value of $f(\phi, p)$ at the $1.1 \times$ $10^{6}$ macroparticle locations. The coherent force was calculated using 600 fine bins and smoothed to obtain 50 statistically independent bins within the bunch. There was no sign of thermalization, probably because the simulation was in one dimension. From Fig. 16 it is clear that Eq. (39) does not yield an exact match, but the two mountain range plots are quite similar even for $\chi$ as small as $1 \%$. Larger values of $\chi$ produced better agreement between the simulations and Eq. (39). This is reminiscent of the coasting beam result in the introduction, where the soliton parameters were close to the self-bunching condition for the density difference.

Comparable simulations have been done using the solutions of the iterative code as initial conditions. The
TABLE I. Simulation parameters for Fig. 15. The number of macroparticles used in the simulation is denoted by $N_{\text {macro }}$, and $N_{\text {bin }}$ is the number of statistically independent longitudinal bins used for the coherent force calculation.

\begin{tabular}{|c|c|c|c|c|c|c|}
\hline Run & $\begin{array}{c}N_{\text {macro }} \\
10^{4} \\
\end{array}$ & $N_{\text {bin }}$ & $A_{0}$ & $a$ & $b$ & $\begin{array}{c}\ell \\
10^{-6} \\
\end{array}$ \\
\hline 1 & 3 & 20 & 0.25 & 0.05 & 0.10 & 5.4 \\
\hline 2 & 3 & 20 & 0.50 & 0.01 & 0.10 & 0.2 \\
\hline 3 & 3 & 20 & 0.75 & 0.10 & 0.03 & -120 \\
\hline 4 & 3 & 20 & 1.00 & 0.05 & 0.10 & 87 \\
\hline 5 & 28 & 40 & 1.25 & 0.10 & 0.05 & -560 \\
\hline 6 & 3 & 20 & 1.50 & 0.05 & 0.10 & 190 \\
\hline 7 & 3 & 20 & 2.00 & 0.05 & 0.10 & 640 \\
\hline
\end{tabular}

iterative code produced solitons that were well matched, providing an additional check to the correctness of the theory. The theory, of course, considers a smooth phase space distribution but actual beams are composed of particles. We go on to consider the long time evolution of these solitons when the discrete nature of the distribution is included.

\section{COLLISIONAL EFFECTS}

The proton bunches in Figs. 2-5 show well-defined solitons. RHIC also accelerates and stores gold ions, but no long-lived solitons have been observed in gold beams. The present section considers a possible reason for this discrepancy. The Coulomb collision rate for a single species plasma is [23]

$$
\nu_{\mathrm{coll}}=\left(\frac{\pi}{2}\right)^{3 / 2 n Z^{4} r_{p}^{2} c^{4}} \frac{A^{2} \sigma_{v}^{3}}{\ln \Lambda}
$$

where $n$ is the number of ions per unit volume, $r_{p}$ is the classical proton radius, $Z$ is the atomic number of the ion, $A$ is the atomic mass, $c$ is the speed of light, $\sigma_{v}$ is the rms velocity, and $\ln \Lambda$ is the Coulomb logarithm. Assuming fixed bunch charge, spatial size, and velocity distribution, the collision rate scales as $Z^{3} / A^{2}$, which is 13 for gold and 1 for protons.

A more sophisticated approach employs the FokkerPlanck equation to describe the evolution of the particle distribution due to this intrabeam scattering [24]. The code assumes that the transverse distributions are Gaussian with equal emittances, and that the longitudinal distribution is a function of action alone. The initial longitudinal distribution was set to be a smooth background with a density enhanced core and a Fokker-Planck solver was used to evolve the system. Figure 17 shows the fraction of the bunch charge remaining in the density enhanced core as a function of time for both gold and protons and for a range of intensities. As one can see from the curves, the gold bunch always smooths out more 

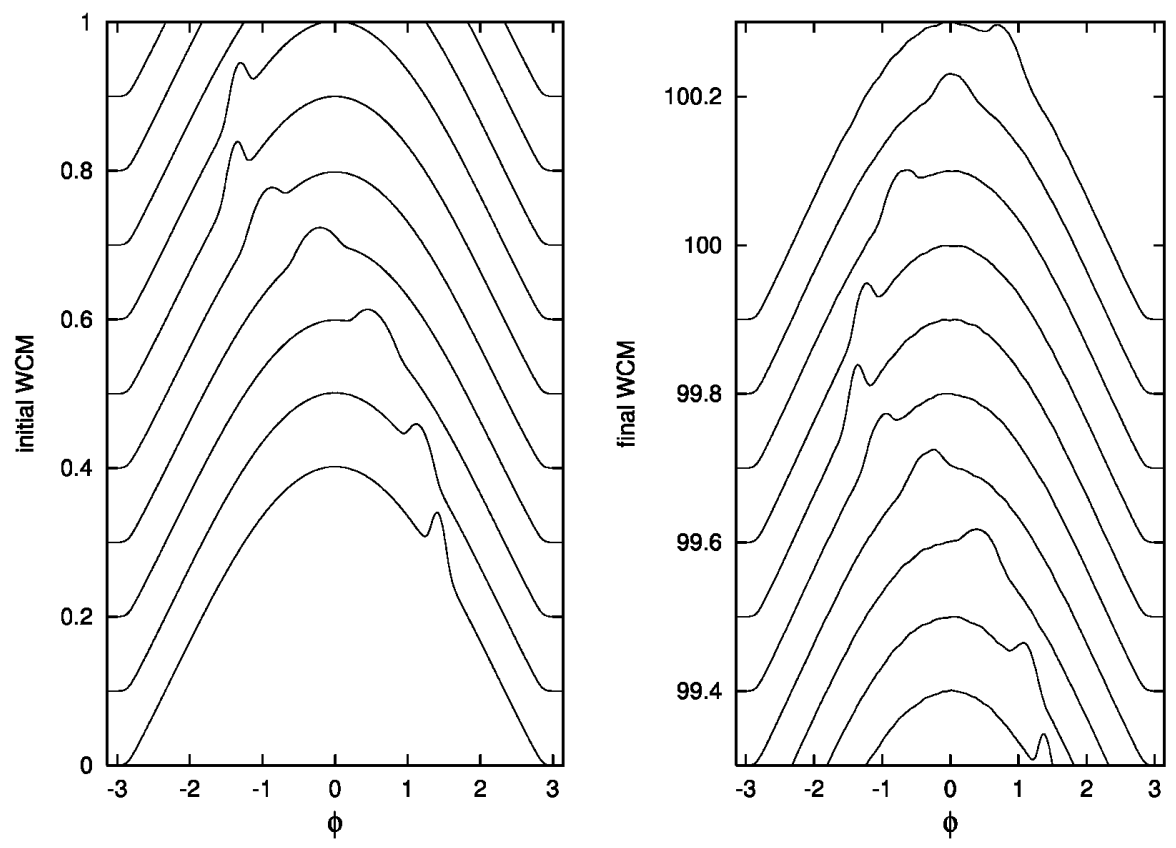

FIG. 16. Simulated wall current monitor data for a soliton with fractional charge $\chi=0.01$. The parameters of the hump were $A_{0}=1, a=0.1$, and $b=0.2$. The intensity parameter was $\ell=0.07$. The plot on the left shows the evolution for the first synchrotron oscillation and the plot on the right shows the evolution over the last of 100 synchrotron oscillations. There are $9 \times 10^{5}$ macroparticles of varying charge and the smoothing length for the coherent force calculation is $1 / 50$ th of the bunch length.

quickly than the proton bunch, and the factor of 13 obtained earlier seems reasonable.

Along with the difference in collision rates the values of $\ell$ were usually quite different for protons and gold, with the proton values being larger by about an order of magnitude. This was predominantly due to the low $\mathrm{rf}$

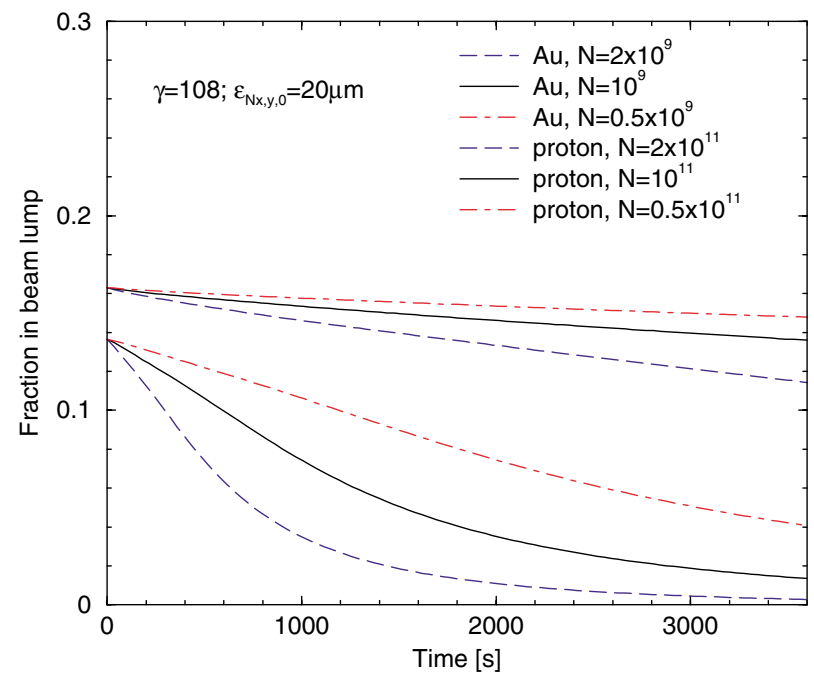

FIG. 17. (Color) Evolution of the fraction of particles in the high density core as a function of time for typical values of Lorentz factor $(\gamma)$ and $95 \%$ normalized transverse emittance $(\epsilon)$ during a RHIC store. For protons the design intensity is $N \approx 10^{11}$ particles per bunch while there are typically $N \approx 10^{9}$ gold ions per bunch. All the proton curves lie above all the gold curves. voltage required for protons, which were injected just above the transition energy. Hence, for a given value of $\chi$, the dimensions of a soliton in a proton bunch would be about twice as large as a soliton with the same $\chi$ in a gold bunch. If we take a simple diffusion model, the rate at which a soliton dissipates will scale as $\nu_{\text {coll }} / a^{2}$, with $a$ being a typical dimension of the soliton. With this extra factor, the dissipation rate for solitons in a gold bunch is about 50 times faster than the dissipation rate for a comparable soliton in a proton bunch. Deuterons have not been studied as carefully as protons, but the data are roughly consistent with this picture.

\section{CONCLUSIONS}

A theoretical framework for understanding stable solitons in bunched beams has been presented. A key result is that a defocusing impedance can lead to humps, while such an impedance can only give holes in coasting beams. Data from RHIC show that humps with a defocusing impedance are present in actual bunches. An analytic formula describing isolated solitons has been presented and verified using numerical simulations. Iterative solutions of the Vlasov equation also agree with simulations so there are three different theoretical approaches that are in agreement with the data.

\section{ACKNOWLEDGMENTS}

We thank J. M. Brennan, S. Koscielniak, and S. Peggs for insightful comments, and F. Severino for developing 
the data acquisition software. M. B.'s and J.W.'s work was supported by the U.S. Department of Energy under Contracts No. DE-AC05-00OR2275 and No. DE-AC0298CH10886. A. L. and H.S. acknowledge the support of the Deutsche Forschungsgemeinschaft, GRK 698: Nonequilibrium phenomena and phase transitions in complex systems.

\section{APPENDIX: SPACE CHARGE FORCES WITH GAUSSIAN BEAMS}

For a round Gaussian beam with rms radius $\sigma$ in a round pipe of radius $b$ the radial electric field is given by

$$
E_{r}(r, z-v t)=\frac{Z_{0} I e^{j \omega(t-z / v)}}{2 \pi \beta r}\left(1-e^{-r^{2} / 2 \sigma^{2}}\right) .
$$

Setting $E_{r}=-\partial \Phi / \partial r$, the leading order term for the longitudinal field is [25]

$$
\begin{aligned}
E_{z}(r, z-v t) & =\frac{-1}{\gamma^{2}} \frac{\partial \Phi}{\partial z} \\
& =\frac{j \omega Z_{0} I}{2 \pi \beta^{2} \gamma^{2} c} \int_{r}^{b} \frac{d r_{1}}{r_{1}}\left(1-e^{-r_{1}^{2} / 2 \sigma^{2}}\right) .
\end{aligned}
$$

The longitudinal impedance per unit length is found by averaging this field over the radial distribution, $Z_{\|}^{\prime}=$ $-\left\langle E_{z}\right\rangle / I$,

$$
\begin{aligned}
Z_{\|}^{\prime}=-\frac{j \omega Z_{0}}{2 \pi \gamma^{2} \beta^{2} c} \int_{0}^{b} \frac{r_{2} d r_{2}}{\sigma^{2}} e^{-r_{2}^{2} / 2 \sigma^{2}} \int_{r_{2}}^{b} \frac{d r_{1}}{r_{1}}\left(1-e^{-r_{1}^{2} / 2 \sigma^{2}}\right) \\
=-\frac{j \omega Z_{0}}{4 \pi \gamma^{2} \beta^{2} c} \int_{0}^{b^{2} / \sigma^{2}} \frac{d x}{x}\left(1-e^{-x / 2}\right)^{2} \\
=-\frac{j \omega Z_{0}}{4 \pi \gamma^{2} \beta^{2} c}\left\{\operatorname { l n } ( b ^ { 2 } / \sigma ^ { 2 } ) \left(1-e^{\left.-b^{2} / 2 \sigma^{2}\right)^{2}}\right.\right. \\
\left.\quad-\int_{0}^{b^{2} / \sigma^{2}} \ln (x) e^{-x / 2}\left(1-e^{-x / 2}\right) d x\right\} \\
\approx-\frac{j \omega Z_{0}}{4 \pi \gamma^{2} \beta^{2} c}\left\{\ln \left(b^{2} / \sigma^{2}\right)-2 \ln 2+\gamma_{E}\right\},
\end{aligned}
$$

where $\gamma_{E} \approx 0.577$ is Euler's constant. Using $Z / n=$ $2 \pi \beta c Z_{\|}^{\prime} / \omega$ and approximating the numerical factors in
Eq. (A6) leads to Eq. (1). The approximation is accurate to better than $1 \%$ for $b / \sigma>3$.

[1] H. Schamel, Phys. Rep. 140, 161 (1986).

[2] H. Schamel, Phys. Rev. Lett. 79, 2811 (1997).

[3] H. Schamel, DESY Report No. 97-161, 1997.

[4] H. Schamel and R. Fedele, Phys. Plasmas 7, 3421 (2000).

[5] S. Koscielniak, S. Hancock, and M. Lindroos, Phys. Rev. ST Accel. Beams 4, 044201 (2001).

[6] J-M. Griessmeier, H. Schamel, and R. Fedele, Phys. Rev. ST Accel. Beams 5, 024201 (2002).

[7] R. A. Dory, Plasma Phys. 6, 511 (1964).

[8] D. Boussard, in Proceedings of the Joint US-CERN Accelerator School, Texas, 1986, Lecture Notes on Phys. Vol. 296 (Springer-Verlag, Berlin, 1987), p. 289.

[9] R. J. Pasquinelli, in Proceedings of the PAC 1995, Dallas, TX (IEEE, Piscataway, NJ, 1995), p. 2379.

[10] L. K. Spentzouris, J.-F. Ostiguy, and P. L. Colestock, Phys. Rev. Lett. 76, 620 (1996).

[11] M. Blaskiewicz, J. M. Brennan, P. Cameron, and J. Wei, in Proceedings of the PAC 2003, Portland, OR (IEEE, Piscataway, NJ, 1993), p. 394.

[12] F. Caspers and D. Mohl, CERN Report No. CERN/PS 98051 (D1), 1998.

[13] Based on F. Sacherer's unpublished notes, courtesy of E. Raka.

[14] H. Schamel and A. Luque (to be published).

[15] V. Lebedev, presentation to the Fermilab Accelerator Advisory Committee, 2003.

[16] M. Blaskiewicz, J. M. Brennan, P. Cameron, W. Fischer, J. Wei, A. Luque, and H. Schamel, in Proceedings of the PAC 2003, Portland, OR (Ref. [11]), p. 3029.

[17] M. Blaskiewicz, J. M. Brennan, P. Cameron, and W. Fischer, in Proceedings of the EPAC 2002, Paris (EPS-IGA/CERN, Geneva, 2002), p. 1488.

[18] H. Goldstein, Classical Mechanics (Addison-Wesley, Philippines, 1980).

[19] F. Sacherer, IEEE Trans. Nucl. Sci. 18, 1105 (1971).

[20] We use the fortran routine RD by B.C. Carlson, E. M. Notis, and R. L. Pexton, www.netlib.org

[21] I. S. Gradshteyn and I. M. Ryzhik, Table of Integrals, Series, and Products (Academic Press, New York, 1980).

[22] C. R. Prior, AIP Conf. Proc. 448, 85 (1998).

[23] See, e.g., S. Ichimaru, Basic Principles of Plasma Physics, A Statistical Approach (Benjamin/Cummings, New York, 1973), Sec. 1.3.

[24] J. Wei, AIP Conf. Proc. 496, 197 (1999).

[25] L. Palumbo and V. G. Vaccaro, CERN Report No. 87-03, 1987 , p. 341. 